\title{
VOLUME ISOTROPIC TURBO SPIN ECHO ACQUISITION MAGNETIC RESONANCE IMAGING PULSE SEQUENCE IN ASSESSMENT OF TEMPOROMANDIBULAR DISORDERS
}

\author{
Mohamed Khalifa Zayet *
}

\begin{abstract}
Background: Magnetic resonance imaging is the chief imaging modality used in assessment of temporomandibular disorders.

Aim of the study: This study aimed to investigate the validity of Volume ISotropic Turbo spin echo Acquisition (VISTA) pulse sequence in the assessment of temporomandibular disorders.

Subjects and Methods: Fifteen patients with temporomandibular joint related complaints performed MRI bilaterally using VISTA proton density and two-dimensional proton density turbo spin echo pulse sequences. Four-point scale was used to assess the clarity of articular disc position, disc morphology, cortical bone delineation, and overall image quality of both tested techniques. Besides, the final diagnosis of each case was obtained.
\end{abstract}

Results: The main and standard deviation of traditionally used 2D proton density images in determination of disc position, disc morphology, delineation of cortical bone and overall image quality were $(2.57 \pm 0.49),(2.37 \pm 0.60),(2.8 \pm 0.4),(2.57 \pm 0.49)$, respectively. While those for VISTA images were $(1.8 \pm 0.4),(1.53 \pm 0.72),(2.1 \pm 0.60),(1.7 \pm 0.64)$ with the same order. The final diagnosis was similar in both techniques in 25 cases out of 30 .

Conclusion: Volume ISotropic Turbo Spin Echo Acquisition (VISTA) magnetic resonance pulse sequence could reveal the temporomandibular joint related structures, but with less image quality than that of traditionally used two-dimensional proton density images.

KEYWORDS: VISTA - pulse sequence - temporomandibular disorders

\section{INTRODUCTION}

Temporomandibular disorders are group of problems related to masticatory muscles and temporomandibular joint (TMJ) ${ }^{(1)}$. These disorders affect significant percent of people ${ }^{(2,3)}$ that may reach $40 \%$ in certain populations. Accordingly, they constitute a burden on clinicians for diagnosing and treatment of such cases ${ }^{(4,5)}$. Radiographic imaging of TMJ is a very crucial element in the triad of diagnosis beside detailed history and

\footnotetext{
* Ass. Professor, Oral and Maxillofacial Radiology, Faculty of Dentistry, Cairo University
} 
through clinical examination, where the ideal imaging modality should be able to reveal hard and soft issue components of the joint ${ }^{(6)}$. Therefore, Magnetic Resonance Imaging (MRI) is considered the gold standard in TMJ imaging because of its unique capability in revealing the articular disc ${ }^{(7)}$, where its position and morphology are considered major imaging findings in the diagnosis of temporomandibular disorders ${ }^{(8)}$. Moreover, magnetic resonance imaging does not stand far away from computed tomography in revealing bony changes in temporomandibular joint ${ }^{(9)}$.

Magnetic resonance imaging as a cross-sectional imaging modality shares its partners; multislice computed tomography and cone beam computed tomography, in their ability to produce images in any of the three orthogonal planes or any orthogonalderived oblique planes ${ }^{(10)}$. However, this ability differs in its algorithm of formation in the three modalities. In both X-ray dependent modalities, it is a matter of post-imaging processing, where the software reconstruct the axial raw data in multislice computed tomography or volumetric raw data in cone beam computed tomography into crosssectional two-dimensional (2D) images in any plane (11). On the other side, MRI depends on intra-imaging algorithm to produce cross-sectional 2D images in the required planes, with different parameters that suit diverse pulse sequences ${ }^{(12)}$.

"Pulse sequences" is a term confined to MRI scans to denote the types of produced images. Multiple pulse sequences families including spin echo, fast (turbo) spin echo, and gradient echo, are available in MRI scanners in order to suit different imaging applications for various body organs ${ }^{(13)}$. Regarding temporomandibular imaging, multiple MRI pulse sequences were investigated over the last decades in order to determine the most appropriate sequence(s) for various diagnostic utilities ${ }^{(14-19)}$. Additionally, three-dimensional (3D) pulse sequences are now available to examine different organs including musculoskeletal systems from different planes ${ }^{(20-23)}$. The chief advantage of tree-dimensional data acquisition is the possibility of reviewing the pathological changes that could occur in imaged structures from different planes and angulations, which augments the diagnostic capabilities of the utilized imaging modality, without significant burden on scanning time.

The aim of this study was to investigate the validity of one of three-dimensional MRI pulse sequences; Volume ISotropic Turbo spin echo Acquisition (VISTA) in diagnosing temporomandibular disorders.

\section{SUBJECTS AND METHODS}

This prospective diagnostic accuracy study was approved by research ethics committee of Faculty of Dentistry, Cairo University. It was conducted on 15 subjects with 30 temporomandibular joints, they were 12 females and 3 males, with age ranged from 17 to 44 years with average 24.7 years. The inclusion criteria were patients with TMJ related signs and symptoms and indicated for MRI scanning; like pain or tenderness in the area of the joint, TMJ sounds, limited mouth opening, or shift in the midline during opening. The exclusion criteria were those who cannot perform MRI scan like those with pacemaker, cerebral aneurysm clips, intra-ocular or periocular foreign body, claustrophobic patients, and pregnant females according to preoperative MRI examination questionnaire ${ }^{(24)}$. Moreover, patients with previous TMJ surgeries or arthrocentesis procedure were also excluded. Written informed consent was taken from each patient after explanation of the research outline, scan procedures, benefits and possible complications, as well as confidentiality of the data, except one illiterate patient, where it was verbal.

MRI scanning: Patients were scanned using 1.5 Tesla superconductive magnet (Philips, Achieva, Netherlands). Scans were performed at Radiodiagnosis outpatient clinic in Kasr El-Einy hospital. 
The investigator was present in all MRI procedures to check the image quality and to instruct the patients what to do during the MRI examination.

MRI scan preparation: Any removable metallic object including removable partial dentures, earrings, necklace or sweater with zipper was removed before the start of the scan. Scout MRI scan was done in the beginning followed by $\mathrm{T} 2$ TSE on which planning of pulse sequences were performed; proton density-turbo spin echo parasagittal cuts were obtained with the following parameters: repetition time (TR): $1500 \mathrm{msec}$, echo time (TE): $30 \mathrm{msec}$, slice thickness: $2.5 \mathrm{~mm}$, inter slice gap: $0.3 \mathrm{~mm}$, FOV: $150 \mathrm{~mm}$, frequency matrix: 256, phase matrix: 256, NEX: 3, Number of slices: 11 , stacks:2, scanning time 2:30 min. with application of TMJ surface coil. 3D imaging VISTA pulse sequence was performed with the following parameters: repetition time (TR): $1500 \mathrm{msec}$, echo time (TE): $30 \mathrm{msec}$, slice thickness: $1 \mathrm{~mm}$, Inter slice gap: $0.5 \mathrm{~mm}$, FOV: $150 \mathrm{~mm}$, Frequency matrix: 256, Phase matrix 256, NEX: 1, stacks: 2 , scanning time 4:35 min with application of TMJ surface coil. Both pulse sequences were done while the patient occluding in centric occlusion then in maximum opening.

\section{Image analysis}

Images were assessed by an oral and maxillofacial radiologist with 17-year experience. The assessment was done with haphazard order of the cases, with Philips DICOM viewer 3.0 software on an 18.5inch monitor (SyncMaster B1930N, Samsung, Seoul, South Korea) with a resolution of $1366 \times 768$. Using image enhancement tools such as zooming, changing the brightness, or contrast was allowed. The assessment session was ceased after 45 minutes maximum or occurrence of eye or mental fatigue. A four-point scale was used to evaluate the quality of the images regarding clarity, visibility, resolution, and delineation as follows: $0=$ Poor images, $1=$ Fair images, $2=$ Good images, $3=$ Very good images .

This four point scale was applied on para-sagittal cuts of both pulse sequences taken in closed position (Figure 1) regarding evaluation of the following: determination of disc position, determination of disc morphology, cortical bone delineation (condyle, glenoid fossa and articular eminence), and the overall quality of the images. Moreover, the final diagnosis of each case, whether it is normal, anterior disc displacement with reduction or anterior disc displacement without reduction, was determined through images of closed and open positions.

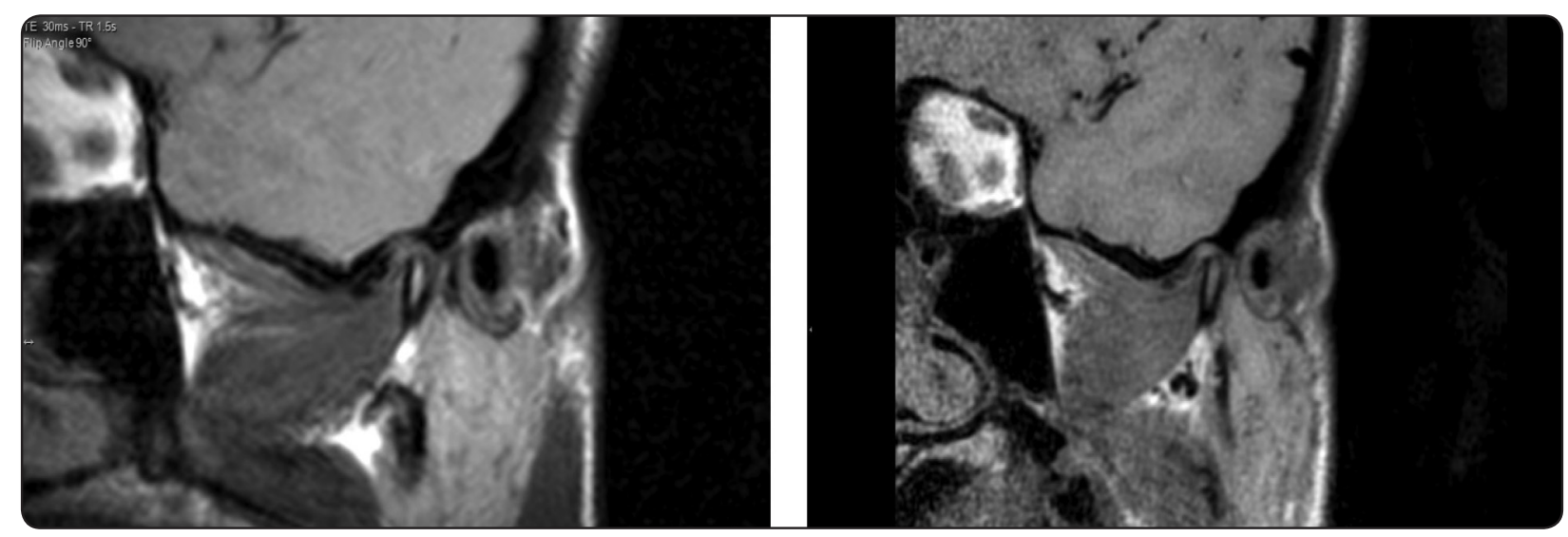

Fig. (1): Para-sagittal proton density turbo spin echo (left) and VISTA proton density (right) in closed position. 


\section{Statistical analysis:}

Data were statistically described in terms of mean \pm standard deviation. Wilcoxon signed rank test for paired (matched) samples was used in comparison between VISTA and 2D proton density images for determination of disc position, disc morphology, delineation of cortical bone, and overall image quality, while weighted Kappa test was used for correlation between final diagnosis obtained through both techniques images. Statistical analyses were carried out using MedCalc software, Belgium.

\section{RESULTS}

In this study VISTA and conventionally used 2D proton density magnetic resonance images were used in assessment of 30 temporomandibular joints. Regarding disc position, in proton density images, the score 3 was obtained in 17 cases and score 2 in 13 cases with mean and standard deviation 2.57 \pm 0.49 . While in VISTA derived images, score 3 was not obtained in any case, score 2 in 24 cases, and six cases got the score 1, with mean and standard deviation $1.8 \pm 0.4$. Wilcoxon test revealed significant difference between them $(p<0.0001)$

For disc morphology, proton density images had score 3 in 13 cases, score 2 in 15 cases, and score 1 in two cases only. While for VISTA, twenty cases had score 2 , six cases for score 1 , and 4 cases for score 0 . The mean and standard deviation for proton density and VISTA images were $2.37 \pm 0.60$ and $1.53 \pm 0.72$, respectively. There was statistically significant difference between both investigated techniques $(p<0.0001)$.

For delineation of TMJ related cortical bone, score 2 was obtained in seven cases only and the remaining cases had score 3 in proton density images with mean and standard deviation $2.8 \pm 0.4$. In the other tested technique, score 3,2 , and 1 were present in 7, 20, 3 cases, respectively. The mean and standard deviation was $2.1 \pm 0.60$. Statistical significant difference was also revealed $(p<0.0001)$.

With respect to the overall image quality, statistical significant difference was present $(p<$ 0.0001 ), where very good images in proton density were 17 cases and good images were 13 cases (mean and standard deviation $2.57 \pm 0.49$ ). on the other side, the score of very good images was not obtained in any case, the majority were good images (25 cases), fair images were in two cases, and three cases had poor overall quality (mean and standard deviation $1.7 \pm 0.64$ ) (Figure 2)

The final diagnosis obtained from closed and open positions images was similar between proton density and VISTA derived images in 25 cases, with correlation coefficient 0.81 and confidence interval $0.65-0.96$.

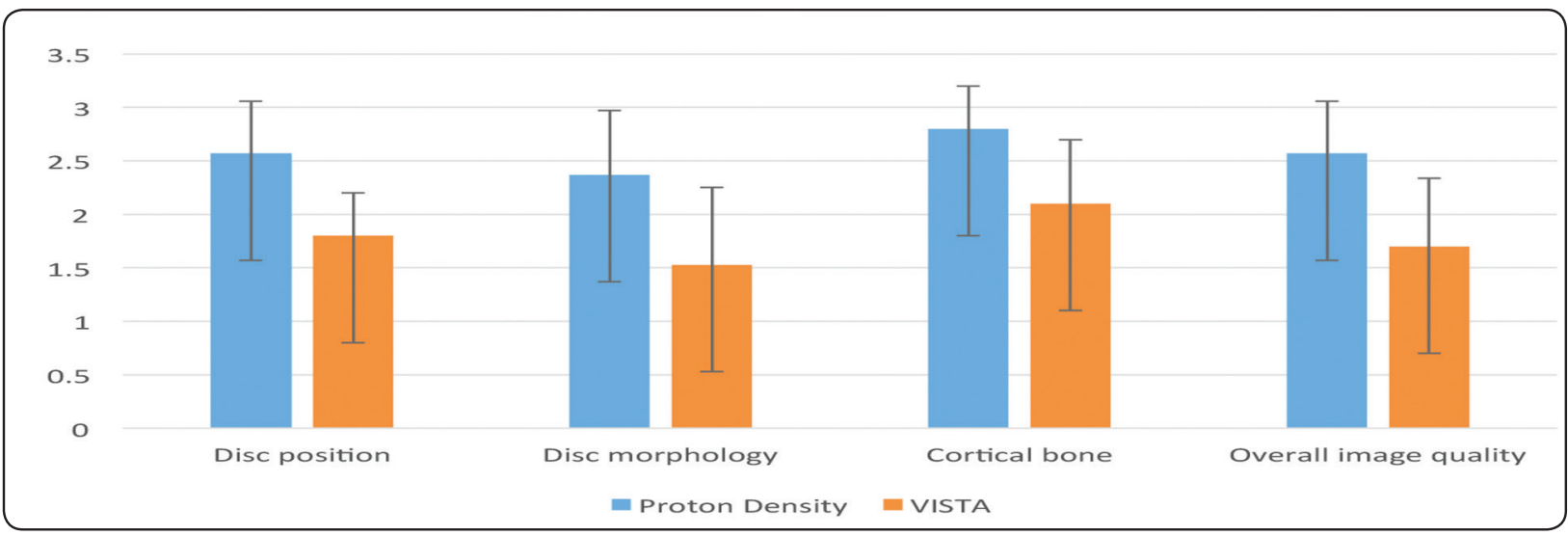

Fig. (2): Bar chart showing the mean of scores given proton density and VISTA images. 


\section{DISCUSSION}

This study was conducted to investigate the appropriateness of VISTA proton density images as one of 3D MRI techniques in diagnosing of temporomandibular disorders and compare its results with those of conventionally used 2D images. The results of the study showed the capability of the test technique in determination of articular disc position and morphology as well as delineation of bony articulating surfaces but with lower image quality than the usual two-dimensional proton density technique.

Determination of articular disc position is the first objective in interpreting of magnetic resonance images of TMJ, As it is considered the chief diagnostic sign for differentiation between both main categories of temporomandibular disorders; intra-capsular and muscular disorders. In the current study, proton density images had the highest score (score 3) in $56.7 \%$ of the cases, while none of VISTA images got that. Visibility of articular disc is not confined to determination of its position, but extends into determination of its morphology, which radically influences the treatment plan and intensely refers to the prognosis of the case. In the present study proton density images scored 3 in $43.3 \%$ of the cases and $50 \%$ scored 2 . On the other side, the highest score provided with VISTA images was score 2 in $66.7 \%$ of the cases.

These results were not similar to those of Sugimori et al $2013^{(25)}$, this difference is due to that they measured the visibility of anterior and posterior bands of the articular disc without focusing on the level of clarity of the structures or the quality of resultant images.

From both previously mentioned results, it could be expected that VISTA-proton density has lower capability on diagnosing of temporomandibular disorder as the diagnosis obtained from VISTA images agreed that of proton density in $83.3 \%$ of cases. Furthermore, statistical analysis showed that correlation coefficient between them was 0.81 and the confidence interval was $0.65-0.96$.

Cortical bone changes, being a common sequel of long standing temporomandibular disorders (26) and a primary feature in cases of osteoarthritis (27), rendered its detection one of the secondary diagnostic findings in MR images. Regarding the delineation of TMJ related cortical bone, there was statistically significant difference between VISTA and traditional 2D proton density images. Where very good images were present in only $23.3 \%$ of VISTA images, compared to $76.7 \%$ of proton density images.

With respect to the overall image quality, that of VISTA was significantly inferior to that of 2D proton density images as the mean score of the former was $2.57 \pm 0.49$, while that of the later was $1.7 \pm 0.64$. The reduced image quality of VISTA images could be attributed to the main drawback of 3D sequences which is the decreased Signal Noise Ratio (SNR) when compared with that of traditional 2D proton density images. The reason for decreased SNR is the thin slice thickness applied in VISTA sequence, which should be $1 \mathrm{~mm}$ or less to obtain raw data suitable for multiplanar reconstruction, where it was $2.5 \mathrm{~mm}$ in proton density images. The other reason is the difference in number of excitations (NEX) which was three folds in 2D proton density sequence.

The main reason for conducting the current study was to save the time of the scans, as extended scanning time is considered one of the main limitations of magnetic resonance imaging. Therefore, any attempt to reduce the scanning time should be done, where 3D data acquisition allows for reconstruction of images in sagittal, coronal, and axial planes, in addition to multiple oblique planes. However, this reduction in time should not jeopardize the quality of the resultant images. Unfortunately, most of factors that could be used to increase SNR and thus improve the image quality 
such as increasing NEX, or using larger slice thickness, or decreasing the acquisition matrix are associated with increasing the time of the scan or reduced spatial resolution ${ }^{(28)}$.

Diagnosis of pathological conditions and differentiation between various diseases depends on quantitative and qualitative assessment of diagnostic tools including radiographic images. Magnetic resonance imaging follows this concept in some of its applications ${ }^{(29,30)}$ including musculoskeletal structures ${ }^{(31)}$, but regarding temporomandibular joint, qualitative assessment is the dominating method. Therefore, the quality of the images is very crucial for proper diagnosis and consequently appropriate treatment planning. Moreover, 3D pulse sequences are recommended to be used in particular practical situations; which are presence of real clinical need for multiplanar reconstruction, with highly specifications MRI scanner, and well trained radiologist.

\section{CONCLUSION}

Volume Isotropic Turbo Spin Echo Acquisition (VISTA) magnetic resonance pulse sequence could reveal the temporomandibular joint related structures, needed for diagnosis of temporomandibular disorders, such as articular disc and cortical bone but with less image quality than that of traditionally used two-dimensional proton density images. Therefore, its usage should be confined to cases where multiple imaging planes are essential for diagnostic tasks.

\section{Conflict of interest:}

The author reports no conflict of interest

\section{ACKNOWLEDGEMENT}

Great appreciation to Mrs. Hala Ali, MRI technician in Kasr El-Einy Hospital, for her valuable efforts in imaging procedures of magnetic resonance scans.

\section{REFERENCES}

1. Telishevska UD, Telishevska OD. Classifications of temporomandibular disorders and patients' examination protocols - comparative analysis by the convenience of their daily use in clinical practice. Wiad Lek. 2018;71(3 pt 2):738-745.

2. da Silva CG, Pachêco-Pereira C, Porporatti AL, Savi MG, Peres MA, Flores-Mir C, Canto Gde L. Prevalence of clinical signs of intra-articular temporomandibular disorders in children and adolescents: A systematic review and meta-analysis. J Am Dent Assoc. 2016 Jan;147(1): 1018.e8.

3. Christidis N, Lindström Ndanshau E, Sandberg A, Tsilingaridis G. Prevalence and treatment strategies regarding temporomandibular disorders in children and adolescents-A systematic review. J Oral Rehabil. 2019 Mar;46(3):291-301

4. Sena MF, Mesquita KS, Santos FR, Silva FW, Serrano KV. Prevalence of temporomandibular dysfunction in children and adolescents. Rev Paul Pediatr. 2013 Dec;31(4):53845. Review.

5. de Melo Júnior PC, Aroucha JMCNL, Arnaud M, Lima MGS, Gomes SGF, Ximenes R, Rosenblatt A, Caldas AF Jr. Prevalence of TMD and level of chronic pain in a group of Brazilian adolescents. PLoS One. 2019 Feb 8;14(2):e0205874.

6. Larheim TA, Hol C, Ottersen MK, Mork-Knutsen BB, Arvidsson LZ. The Role of Imaging in the Diagnosis of Temporomandibular Joint Pathology. Oral Maxillofac Surg Clin North Am. 2018 Aug;30(3):239-249.

7. Talmaceanu D, Lenghel LM, Bolog N, Hedesiu M, Buduru S, Rotar H, Baciut M, Baciut G. Imaging modalities for temporomandibular joint disorders: an update. Clujul Med. 2018 Jul;91(3):280-287. Review.

8. Renton T, Durham J, Aggarwal VR. The classification and differential diagnosis of orofacial pain. Expert Rev Neurother. 2012 May;12(5):569-76.

9. Sinha VP, Pradhan H, Gupta H, Mohammad S, Singh RK, Mehrotra D, Pant MC, Pradhan R. Efficacy of plain radiographs, CT scan, MRI and ultrasonography in temporomandibular joint disorders. Natl J Maxillofac Surg. 2012 Jan;3(1):2-9.

10. White, S. C. and Pharoah, M. J. (2014) Oral radiology : Principles and Interpretation. $7^{\text {th }}$ ed. Elseiver Mosby. 
11. Dillenseger JP, Matern JF, Gros CI, Bornert F, Goetz C, Le Minor JM, Constantinesco A, Choquet P. MSCT versus CBCT: evaluation of high-resolution acquisition modes for dento-maxillary and skull-base imaging. Eur Radiol. 2015 Feb;25(2):505-15.

12. Foltz WD, Jaffray DA. Principles of magnetic resonance imaging. Radiat Res. 2012 Apr;177(4):331-48. Review.

13. Bitar R, Leung G, Perng R, Tadros S, Moody AR, Sarrazin J, McGregor C, Christakis M, Symons S, Nelson A, Roberts TP. MR pulse sequences: what every radiologist wants to know but is afraid to ask. Radiographics. 2006 Mar-Apr;26(2):513-37. Review.

14. Schellhas KP, Wilkes CH. Temporomandibular joint inflammation: comparison ofMR fast scanning with T1- and T2-weighted imaging techniques. AJR Am J Roentgenol. 1989 Jul;153(1):93-8.

15. Bell KA, Jones JP, Miller KD, al-Refal D. The added gradient echo pulse sequence technique: application to imaging of fluid in the temporomandibular joint. AJNR Am J Neuroradiol. 1993 Mar-Apr;14(2):375-81.

16. Honda E, Sasaki T, Simm FC, Maruyama K. An optimized fast protocol for magnetic resonance imaging of the temporomandibular joint. Dentomaxillofac Radiol. 2001 Mar;30(2):126-30.

17. Sano T, Widmalm SE, Yamamoto M, Sakuma K, Araki K, Matsuda Y, Okano T. Usefulness of proton density and T2weighted vs. T1-weighted MRI in diagnoses of TMJ disk status. Cranio. 2003 Oct;21(4):253-8.

18. Carl M, Sanal HT, Diaz E, Du J, Girard O, Statum S, Znamirowski R, Chung CB. Optimizing MR signal contrast of the temporomandibular joint disk. J Magn Reson Imaging. 2011 Dec;34(6):1458-64.

19. Cassetta M, Barchetti F, Pranno N, Marini M. Comparing proton density and turbo spin echo $\mathrm{T} 2$ weighted static sequences with dynamic half-Fourier single-shot TSE pulse sequence at 3.0 T in diagnosis of temporomandibular joint disorders: a prospective study. Dentomaxillofac Radiol. 2014;43(3):20130387.

20. Naraghi A, White LM. Three-dimensional MRI of the musculoskeletal system. AJR Am J Roentgenol. 2012 Sep;199(3):W283-93. Review.

21. Tanitame N, Tanitame K, Awai K. Clinical utility of optimized three-dimensional T1-, T2-, and T2*-weighted sequences in spinal magnetic resonance imaging. Jpn J Radiol. 2017 Apr;35(4):135-144.

22. Koontz NA, Wiggins RH 3rd, Mills MK, McLaughlin MS, Pigman EC, Anzai Y, Shah LM. Less Is More: Efficacy of Rapid 3D-T2 SPACE in ED Patients with Acute Atypical Low Back Pain. Acad Radiol. 2017 Aug;24(8):988-994.

23. Chang AL, Yu HJ, von Borstel D, Nozaki T, Horiuchi S, Terada Y, Yoshioka H. Advanced Imaging Techniques of the Wrist. AJR Am J Roentgenol. 2017 Sep;209(3):497510. Review.

24. Shellock FG, Crues JV. MR procedures: biologic effects, safety, and patient care. Radiology. 2004 Sep;232(3):63552. Review.

25. Sugimori Y, Tanaka S, Nishimura T, Yamamoto A, Ohfuji S, Naito Y, Katsumata Y, Miki Y. Usefulness of dual echo volumetric isotropic turbo spin echo acquisition (VISTA) in MR imaging of the temporomandibular joint. Magn Reson Med Sci. 2013 Dec 25;12(4):249-59.

26. Kowalchuk RM, Kowalchuk RO, Kaplan-List K, Caplash JM, Block P. Temporomandibular Joint Internal Derangement Score (TIDS): novel magnetic resonance imaging assessment score and its relation to invasive treatment in patients with clinical temporomandibular joint pathology. Heliyon. 2018 Nov 11;4(11):e00916.

27. Vimort JB, Ruellas A, Prothero J, Marron JS, McCormick M, Cevidanes L, Benavides E, Paniagua B. Detection of bone loss via subchondral bone analysis. Proc SPIE Int Soc Opt Eng. 2018 Feb;10578. pii: 105780Q.

28. Tsao J. Ultrafast imaging: principles, pitfalls, solutions, and applications. J Magn Reson Imaging. 2010 Aug;32(2):25266. doi: 10.1002/jmri.22239. Review.

29. Tognarelli JM, Dawood M, Shariff MI, Grover VP, Crossey MM, Cox IJ, Taylor-Robinson SD, McPhail MJ. Magnetic Resonance Spectroscopy: Principles and Techniques: Lessons for Clinicians. J Clin Exp Hepatol. 2015 Dec;5(4):320-8.

30. Taffel MT, Johnson EJ, Chandarana H. Diffusion Quantification in Body Imaging.Top Magn Reson Imaging. 2017 Dec;26(6):243-249.

31. de Mello R, Ma Y, Ji Y, Du J, Chang EY. Quantitative MRI Musculoskeletal Techniques: An Update. AJR Am J Roentgenol. 2019 Apr 17:1-10. 